\title{
PREVENIR EN SALUD DESDE LA PARTICIPACIÓN: ENFOQUE COMUNICATIVO PARA EL CAMBIO SOCIAL EN ADOLESCENTES CUBANOS ${ }^{1}$
}

\author{
Health prevention from participation; communicative approach for social \\ change in Cuban teenagers
}

\author{
Maitié Rodríguez Wong, Neivys Machado Flores ${ }^{2}$, Grether Pérez Paulete, Alexander \\ Casa Montejo* \\ Universidad Central "Marta Abreu" de Las Villas. Santa Clara, Cuba \\ *Unidad Provincial de Propaganda Villa Clara. Santa Clara, Cuba
}

\section{Resumen}

El presente proyecto engloba 4 investigaciones realizadas en la Escuela de Conducta "Roberto Ambrosio Zamora Machado" de Ciego de Ávila con el objetivo de proponer, desde la participación de los sujetos, estrategias de comunicación para la prevención en salud de las ITS-VIH/SIDA y el tabaquismo en menores internos en dicha institución educativa. Adscritos a la metodología cualitativa, desde la investigación-acción-participación, se utilizan la revisión de documentos oficiales, la entrevista en profundidad, la observación participante y las sesiones en profundidad. La selección de la muestra se corresponde con informantes claves y grupos gestores. Mientras, las categorías de análisis utilizadas son necesidades comunicativas y estrategia de comunicación para la prevención. A partir de la triangulación metodológica, de fuentes y técnicas se obtiene como principales resultados el logro de un cambio a nivel cognitivo y motivacional en los sujetos protagonistas de la investigación desde los que emergen estrategias de comunicación con la finalidad de solucionar sus problemáticas de salud. A partir de los resultados obtenidos se recomienda extender esta experiencia a instituciones educacionales similares en el resto del país.

Palabras clave: Comunicación en salud, estrategias, prevención

\section{Abstract}

The current project encompasses four investigations carried out in the Behavioral School "Roberto Ambrosio Zamora Machado" in Ciego de Ávila. Its aim is to propose communication strategies from the participation of the subjects, to prevent STD/HIVAIDS and tobacco consumption in under aged in such educational institution. It is used in this research the revision of official documents, the interview, the observation of the participants and sessions with groups. All of these techniques are applied with

\footnotetext{
1 Este artículo sistematiza los resultados del trabajo de campo para las Tesis de Diploma de las licenciadas Katerin García Márquez (2014), Taymara Varona Morales (2015) y Arianna Sánchez Hurtado (2015).

22 Autor de contacto: Neivys Machado Flores neivys@uclv.cu
} 
a qualitative methodology, from the investigation-action-participation method. The sample is composed of key informants and developing groups. Besides, the analysis categories used constitute communicative needs and communicative strategy for prevention. The achievement of a change to the cognitive and motivational level in the protagonist subjects of the investigation was obtained from the methodological triangulation and also from the triangulation of sources and techniques. Communication strategies emerge from these subjects with the objective of solving their health problems. From the results obtained it is recommended to broaden this experience to similar educational institutions throughout the country

Keywords: Communication in health area, strategies, prevention

\section{Cómo citar el artículo}

Rodríguez Wong, M., Machado Flores, N., Pérez Paulete, G., Casa Montejo, A. (2017). Prevenir en salud desde la participación: enfoque comunicativo para el cambio social en adolescentes cubanos. Revista de Comunicación y Salud, vol. 7, pp.

\section{INTRODUCCIÓN}

La promoción y la prevención, son dos conceptos que se utilizan indistintamente de manera equívoca para referirse a un mismo fenómeno. "Pese a que a menudo se produce una superposición del contenido y de las estrategias, la prevención se define como una actividad distinta a la promoción de la salud" (OMS, 1998, citado en Redondo, 2004, p. 15).

Su principal diferencia radica en su enfoque: la promoción trabaja con población sana, la prevención con población enferma o en riesgo de enfermar (Redondo, 2004). Así, debido a los objetivos y a las características del escenario de investigación, el presente artículo sistematiza una experiencia enmarcada desde prevención en salud.

La importancia de este proceso es avalada por investigaciones, programas y proyectos a nivel mundial, adecuándose a las disímiles características de los diferentes países y regiones. En Cuba, la prevención en salud constituye una prioridad del sistema de salud, en correspondencia con la opinión de Dr. Ricardo González (2012).

En función de ello y del escenario de investigación, este artículo se enmarca en la prevención en salud en adolescentes internos - los que provienen de familias y ambientes sociales disfuncionales- por necesidades educativas especiales y comportamiento social incorrecto en la Escuela de Conducta "Roberto Ambrosio Zamora Machado" de la provincia Ciego de Ávila en la región central del país.

Esta institución educativa de conjunto con el Ministerio del Interior (MININT) cubano demandan acciones de intervención para prevenir las enfermedades de trasmisión sexual en adolescentes con conductas sexuales promiscuas. Igualmente, resulta preciso enfocar la educación en salud hacia la prevención del tabaquismo en esta misma población. 
Los principales antecedentes de esta experiencia en la región central cubana se ubican, desde el ámbito académico, en investigaciones impulsadas por la Universidad Central "Marta Abreu" de Las Villas (UCLV) a partir del trabajo de las facultades de Ciencias Sociales, Humanidades y el Centro de Bienestar Universitario $(\mathrm{CBU})^{3}$. Igualmente, se tuvieron en cuenta los programas sobre la prevención de estas temáticas, desarrollados por la atención primaria de salud y la institución educativa, objeto de investigación.

Con una perspectiva comunicativa- educativa se trabaja con los adolescentes internos en dicha institución educativa para contribuir a la prevención del consumo de tabaco y de las enfermedades de trasmisión sexual en este grupo de riesgo. A partir de las experiencias personales de los adolescentes, entre los 14 y los 15 años, se diseñan, implementan y evalúan estrategias de comunicación para lograr un cambio social y cognitivo, en los protagonistas de la investigación.

Estas estrategias de comunicación de bien público para la prevención identificaron las necesidades comunicativas como primera categoría de análisis para proponer las estrategias comunicativas para la prevención. Desde lo anterior para la intervención en el contexto de investigación, los investigadores se proponen como interrogante de investigación: ¿Cómo contribuir a la prevención de las ITS-VIH/ SIDA y el tabaquismo en menores internos en la Escuela de Conducta de Ciego de Ávila?

Esta interrogante es resuelta a partir de la realización de cuatro investigaciones. Dos de ellas, tributan a la prevención de las ITS-VIH/SIDA y las restantes a la prevención del tabaquismo. En su totalidad responden a los siguientes objetivos específicos:

- Identificar las necesidades comunicativas que sobre las ITS-VIH/ SIDA y el tabaquismo poseen los menores internos en la Escuela de Conducta de Ciego de Ávila.

- Diseñar estrategias de comunicación para la prevención de las ITS-VIH/ SIDA y el tabaquismo en menores internos en la Escuela de Conducta de Ciego de Ávila.

- Implementar las estrategias de comunicación para la prevención de las ITSVIH/ SIDA y el tabaquismo en menores internos en la Escuela de Conducta de Ciego de Ávila.

- Evaluar las estrategias de comunicación para la prevención de las ITS-VIH/ SIDA y el tabaquismo en menores internos en la Escuela de Conducta de Ciego de Ávila.

La intervención realizada propició un importante eslabón para complementar el trabajo realizado por la institución objeto de estudio en función de la educación de estos adolescentes. Demostró la pertinencia de la comunicación como fuente de

\footnotetext{
${ }^{3}$ Este centro se encuentra adscrito al Departamento de Psicología de la UCLV. Su misión es contribuir a fomentar la calidad de vida y la formación integral de los estudiantes, profesores y trabajadores de la comunidad universitaria, con énfasis en la atención psicológica, la promoción de estilos de vidas saludables, la detección de comportamientos riesgosos tanto en el contexto individual como grupal.
} 
cambios sociales, potenciando el papel de los sujetos implicados como agentes de dicho cambio.

\section{Metodología}

Teniendo en cuenta la perspectiva cualitativa de investigación se asume como método la investigación-acción-participación. Su utilización está determinada por la intención de propiciar un cambio social, transformar la realidad y que los sujetos tomen conciencia de su papel en ese proceso de transformación (Sandín, 2003, citado por De la Cobra, 2012).

El proyecto trabaja con informantes claves y con dos grupos gestores. Los informantes claves quedaron conformados por la psicóloga, la psicopedagoga del Órgano de Atención a Menores de la provincia y el director de la Escuela de Conducta. Por su parte, la conformación de los grupos gestores obedece a la inclusión de aquellos actores estratégicos que diseñan, implementan y evalúan las estrategias de comunicación.

El primer grupo gestor está integrado por seis menores internas por comportamiento sexual promiscua, la psicóloga, la psicopedagoga y cuatro auxiliares educativas de la institución educacional para un total de doce personas. Mientras, el segundo grupo gestor se conforma por 14 personas, 8 menores adictos al tabaco y los profesionales antes mencionados. Estos grupos gestores funcionarán como futuros promotores de salud en el contexto donde se desempeñan.

Se trabaja con dos categorías de análisis fundamentales necesidades comunicativas y estrategias de comunicación para la prevención.

La primera categoría es sistematizada por los autores DrC. Osana Molerio Pérez y el Lic. Roeldys González Laffita en el 2011 e incluye subcategorías de análisis como nivel de conocimiento, necesidades de información sobre la temática, recursos comunicativos y divulgación sobre la temática.

Mientras que la categoría estrategia de comunicación para la prevención se construye a partir de las subcategorías propuestas por el DrC. Kirk Díaz Guzmán Corrales y el enfoque de Guiofantes en 1996. Ella propone un modelo de 5 fases para el diseño e implementación de estrategias de comunicación de bien público para la prevención en salud.

En dicho modelo para estrategias comunicativas se explicita la definición del público objetivo, la exploración de acciones comunicativas realizadas con anterioridad, el establecimiento de los objetivos a alcanzar, la definición de los ejes psicológicos conceptuales y líneas de mensajes a difundir, la propuesta creativa de la estrategia que encierra el diseño de soportes comunicativos gráficos y audiovisuales, la elaboración del presupuesto y la implementación y evaluación de las estrategias comunicativas.

Para el desarrollo de estas investigaciones se emplearon como principales técnicas de recogida de datos la revisión bibliográfica de documentos oficiales, la 
observación participante, la entrevista en profundidad y las técnicas de dinámicas de grupo.

El proceso de intervención para la prevención en salud se desarrolla en el período comprendido entre septiembre de 2013 a junio de 2015. Entre 2013 y 2014 se aplicaron las técnicas para los diagnósticos y diseño de las propuestas de estrategias de comunicación. Mientras que de septiembre de 2014 a junio de 2015 se implementaron ambas estrategias. El proceso de evaluación se efectuó durante todo el período de investigación.

La evaluación de la factibilidad de las estrategias comunicativas para el logro del cambio social en los grupos de riesgo se realiza a partir de la aplicación de entrevistas grupales para la medición de los niveles de conocimiento en todo el proceso de intervención. Mientras que los cambios de concientización y conductual se constatan con la determinación de los niveles de participación de los sujetos en las acciones propuestas.

José Luis Rebellato (2000) establece tres niveles de participación en función de la implicación de los sujetos en un determinado proceso: formar parte, tener parte y tomar parte. Según este autor, la idea de participación se completa con este último nivel donde los protagonistas están completamente incluidos en el proceso de toma de decisiones, y son los encargados de planificar y realizar las acciones, lo que sustenta un sentido de pertenencia y concientización con el proyecto del que son partícipes. Así, estos niveles permiten valorar el cambio conductual en los adolescentes cuando alcanzan este tercer nivel de participación a partir de su implicación en el proceso.

La salida del campo se produce sustentada en el criterio de saturación de la información a través de la triangulación de datos, fuentes y técnicas y el análisis de contenido categorial.

\section{LA PREVENCIÓN EN SALUD DESDE LA PARTICIPACIÓN: PRINCIPALES RESULTADOS}

La presentación de los resultados se realizará teniendo en cuenta las etapas en las que se desarrolló el proceso de intervención para la prevención en salud de las ITS-VIH/SIDA y el tabaquismo: la fase diagnóstica y la fase de diseño, implementación y evaluación de las estrategias propuestas.

\subsection{Diagnóstico de las necesidades comunicativas}

Como resultado de la fase diagnóstica con ambos grupos gestores se constata los niveles de conocimientos que poseen sobre las temáticas, las necesidades de información que requieren y los recursos comunicativos para la divulgación de dichas temáticas en el escenario donde conviven. Ello es posible a partir de la realización de técnicas participativas que propician el reconocimiento de los problemas de salud que presentan y la identificación de una posible solución desde la comunicación para dichas problemáticas. 
El desarrollo de las sesiones grupales y las entrevistas en profundidad desvelaron que el conocimiento que poseían los menores internos sobre las temáticas inicialmente era insuficiente. Lo que se justifica por el desconocimiento de varias de las consecuencias provocadas por el hábito de fumar, los beneficios del cese tabáquico, los mitos asociados al consumo del tabaco, las vías para afrontar los síntomas de abstinencias y los daños provocados a la salud por los compuestos químicos.

En este sentido, el otro grupo gestor desconoce términos como falo, vagina, secreciones vaginales, relaciones sexuales sin riesgo y el no dominio de los tipos de ITS y la correspondiente confusión de los síntomas que produce cada uno de ellas.

De manera general, los canales para la divulgación de la temática identificados por los menores lo constituyen las charlas educativas desarrolladas por las instructoras educativas. Afirman que estas charlas son empleadas en la escuela como la principal vía para obtener la información sobre los temas relacionados con la salud.

Estos grupos gestores no son receptores de los mensajes emitidos por los medios de comunicación nacionales, provinciales y municipales debido a la inexistencia de recursos receptores en la institución educativa. Además plantean que no reciben orientación sobre estas temáticas por parte de los padres o tutores legales.

Sin embargo, manifiestan que prefieren recepcionar mensajes sobre temáticas de salud a través de audiovisuales (vídeo-conferencias), afiches, sueltos y plegables. La experiencia investigativa de este proceso interventivo sistematiza además, la preferencia de canales directos para la obtención de información a partir de las dinámicas grupales.

La confrontación de criterios entre el personal entrevistado y las diferentes sesiones con los grupos gestores constata la insuficiencia de los recursos comunicativos para divulgar dichas temáticas. Ello principalmente debido a su inexistencia en la escuela y a la escasez de aquellos provenientes de los programas nacionales.

El nivel de conocimiento inicial que poseían los menores se transformó a partir de la experiencia del proceso desde insuficiente hacia suficiente. En la medida en que avanzaban las sesiones, los grupos gestores comenzaron a profundizar y a motivarse por elevar su conocimiento hacia las temáticas.

La experiencia participativa propicia que los menores comiencen a reconocer el consumo del tabaco y las enfermedades de transmisión sexual como problemas de salud que presentan. Ello permite, a su vez, que se impliquen en la búsqueda de una posible solución.

Además reconocen la necesidad de incluir a los padres como parte de la experiencia. Lo anterior evidencia el cambio cognitivo y motivacional en esta primera fase de la investigación. 


\subsection{Diseño, implementación y evaluación de las estrategias de comunicación para la prevención en salud}

Para el diseño, implementación y evaluación de las estrategias se continuó la lógica participativa donde el sujeto es el protagonista del proceso. En las sesiones grupales se construyeron las distintas fases de la estrategia incluyendo el modelaje de la propuesta creativa, materializada de conjunto con un diseñador gráfico.

Como parte del diseño de las estrategias, los grupos gestores conformaron los objetivos de comunicación, el público objetivo, los ejes psicológicos, las líneas de mensajes y las acciones. Estos elementos diseñados de conjunto con comunicadores sociales fueron implementados posteriormente por los sujetos integrantes de los grupos gestores.

Las estrategias de comunicación fueron presupuestadas por el Órgano de Atención a Menores de Ciego de Ávila, perteneciente al Ministerio del Interior de conjunto con la escuela. Las labores de impresión, y premios fueron financiadas por ambas instituciones.

El público objetivo de ambas estrategias coincide. Se segmenta en los menores internos en la institución educativa y en los padres o tutores legales de dichos menores. Este último segmento de público es incluido a partir de la decisión de los menores pertenecientes a los grupos gestores de involucrar a sus padres en las acciones propuestas.

Igualmente, se decide en los grupos enfocar la comunicación hacia el logro de una concientización sobre la necesidad de cuidar tanto su salud como la de los demás menores internos. Así, como objetivo general de comunicación se proponen:

- Contribuir a la prevención de las ITS-VIH/ SIDA y del tabaquismo en menores internos en la Escuela de Conducta de Ciego de Ávila.

De igual manera, las sesiones grupales con los grupos gestores posibilitaron la construcción de los siguientes objetivos específicos de comunicación:

- Informar a los públicos objetivos acerca de sus necesidades comunicativas sobre ambas temáticas.

- Persuadir a los públicos objetivos sobre la necesidad de disminuir el consumo de tabaco y fomentar conductas sexuales responsables.

- Sensibilizar a los públicos objetivos sobre el peligro que representan las enfermedades de transmisión sexual y el hábito de fumar para la salud.

Desde estos objetivos, emerge como eje psicológico a transmitir en ambas estrategias: fomentar la responsabilidad de los menores internos en la preservación del cuidado de su salud, la seguridad y el bienestar social en la Escuela Conducta de Ciego de Ávila. 
Los grupos gestores deciden que las principales temáticas a tratar en los mensajes se sintetizan en las consecuencias del cigarro, los beneficios del cese tabáquico, los mitos asociados al consumo del tabaco, las vías para afrontar los síntomas de abstinencias, los daños que provocan a la salud los compuestos químicos del cigarro, las infecciones de transmisión sexual, la clasificación y los síntomas que producen y las medidas para la prevención de ellas. Es decir, aquellas temáticas reconocidas en la fase diagnóstica como desconocidas para ellos.

Los elementos comunicativos como el slogan, la gama cromática, el identificador gráfico y los productos comunicativos también emergieron de las necesidades comunicativas de los grupos gestores.

El slogan de la estrategia para la prevención de las ITS-VIH/SIDA es "Protejamos nuestra felicidad". Su elaboración estuvo sustentada en las palabras identificadas por los menores con la frase "sexo seguro".

Por su parte, identifican "Lucha por ti", con el slogan de la estrategia para la prevención del consumo del tabaco cuya confección responde a las palabras identificadas por el grupo gestor sobre la representación social que tienen acerca del "cigarro". Ambas propuestas incitan al cuidado de su salud a partir del fomento de acciones para incorporar estilos de vida más saludables.

La construcción de los identificadores gráficos de conjunto con el diseñador se relaciona con los slogans propuestos. El grupo gestor decide que el primer identificador constituya una síntesis formal del condón masculino, cuya presentación de forma circular trasmite idea de inclusión y participación. Mientras que para la segunda estrategia proponen la unión de elementos como el cigarro, la guadaña y una señal de tránsito, que refleja la prohibición de fumar.

Los grupos gestores construyen la gama cromática a partir de la identificación del rosado y el gris para la estrategia de VIH/SIDA. Ello enfocado en la identificación grupal con estos colores a partir de la asociación del rosa con la identidad de género femenina y el gris con la seriedad y responsabilidad con que deben asumirse las relaciones sexuales.

Mientras que la otra estrategia deciden regirla cromáticamente por los colores rojo y blanco. Dichos colores son percibidos por el grupo como prohibición y peligro en correspondencia con los signos reconocidos a escala mundial con la temática del consumo de tabaco.

Las acciones para la estrategia construidas en ambos grupos gestores son segmentadas en tres etapas: informativa, persuasiva y de recordación. Entre ellas se encuentran las charlas educativas interactivas, los talleres vivenciales, los cine debates, las terapias florales para disminuir la ansiedad de fumar, talleres con padres y representantes legales (aprovechando las reuniones de padres), concursos, juegos de participación y encuentros de conocimientos.

La fase de implementación de las estrategias abarca el período correspondiente a un curso escolar en Cuba (septiembre a junio). En este período ambos grupos gestores comienzan a desarrollar las acciones comunicativas propuestas. Se 
convierten en promotores de salud dentro de su centro educativo y evidencian un cambio motivacional y de concientización en hábitos de vida, asumiendo estilos de vida más saludables.

Al concluir la implementación y, como parte del proceso de evaluación de las estrategias, se confirma que los menores alcanzan un nivel suficiente de conocimiento en ambas temáticas. De igual forma expresan su deseo de implicarse en otras actividades de prevención en salud, manifestando la importancia de tomar parte en proyecto interventivo desde la óptica participativa.

Dicha implementación demuestra la participación real de los grupos gestores, lo que significa un cambio a nivel de conciencia en los adolescentes. Ellos logran una identificación y un sentido de pertenencia en el desarrollo de todas las acciones de las estrategias.

\section{CONCLUSIONES}

El diagnóstico de las necesidades comunicativas que sobre ambas temáticas poseen los grupos gestores demuestra el insuficiente nivel de conocimiento que inicialmente poseen estos adolescentes sobre ellas, lo que incide en que se conviertan en un grupo de riesgo propicio para contraer enfermedades de trasmisión sexual o convertirse en fumadores activos. Dicho diagnóstico justifica la propuesta de estrategias de comunicación para la prevención en salud.

Las estrategias de comunicación de bien público para la prevención se diseñaron a partir de las demandas, prioridades y necesidades comunicativas de ambos grupos gestores para extender estas herramientas hacia el resto de los adolescentes de la escuela.

La implementación de las estrategias posibilitó la evolución desde insuficiente hasta suficiente del nivel de conocimiento así como una concientización en los menores internos a partir del alcance del tercer nivel de participación. Esta experiencia demuestra la función educadora de la comunicación en tanto empodera a los sujetos en pos de la resolución de sus problemáticas cotidianas.

La comunicación para el cambio social desde este proceso afianza la importancia y el papel de los sujetos como agentes de su propio cambio. Constituye un enfoque donde el "diálogo" y la "acción colectiva" trabajan en conjunto para producir cambios sociales. Esta tendencia sitúa a la comunicación como una vía que genera el diálogo horizontal y promueve la participación e implicación de los sujetos para transformar una realidad en conflicto. De ahí, que se destaque como principal reto replicar el proyecto en otras instituciones educativas con características similares o con grupos de riesgos con iguales características sociopsicológicas.

\section{BIBLIOGRAFÍA}

Álvarez, M. (2008). Estrategia de comunicación educativa sobre prevención del VIH/SIDA en estudiantes de medicina del I.S.C.M de La Habana. (Tesis de maestría). Universidad de La Habana, Ciudad de la Habana, Cuba. 
Beltrán, L .R. (2005). La comunicación para el desarrollo en Latinoamérica: un recuento de medio siglo. Documento presentado al III Congreso panamericano de la Comunicación, Buenos Aires, Argentina.

Calviño, M. (1998). Trabajar en y con grupos. Experiencias y reflexiones básicas. La Habana: Editorial Academia.

Carvajal, C. y Torres, A. (2007). Promoción de la Salud en la Escuela Cubana. La Habana: Editorial Pueblo y Educación.

Chernerw, M.; Sanford, J. y Fendrick, M. (2015). Reconciling Prevention and Value in the Health Care System. Recuperado de htpp://helthaffairs.org/blog/2015/03/11/Reconciling Prevention and Value in the Health Care System.

De la Cobra, F. (2012). Valores proambientales en la comunidad Zona 37 de Santa Clara. (Tesis de Licenciatura). Universidad Central "Marta Abreu" de Las Villas, Santa Clara, Villa Clara, Cuba.

Díaz-Guzmán, K. (2009). Fundamentos de Publicidad para comunicadores sociales. Colombia: Editorial Crucet \& Asociados.

García, K. (2014). Estrategia de comunicación para la prevención de ITS-VIH/SIDA en menores internos en la Escuela de Conducta de Ciego de Ávila por comportamiento sexual desordenado. (Tesis de licenciatura). Universidad Central "Marta Abreu" de Las Villas, Santa Clara, Villa Clara, Cuba.

González, R. (2011). Vida sin Humo: Software Educativo para potenciar la prevención del tabaquismo en la Universidad Central "Marta Abreu" de las Villas. (Tesis de Licenciatura). Universidad Central "Marta Abreu" de Las Villas, Santa Clara, Villa Clara, Cuba.

González, R. (2012). Misión: Rescate de Adictos. La Habana: Ediciones Abril.

Guiofantes, S. (1996). Salud y prevención desde el punto de vista psicológico. En Salud Laboral y Ciencias de la Conducta, pp. 25-37.

Gumucio, A. (2002). Haciendo olas: comunicación participativa para el cambio social. Informe para la Fundación Rockefeller. Recuperado de http://www.comminit.com/es/node/150011.

López, F. J. (2008). Participación comunitaria y diagnóstico de necesidades. Recuperado de http://querespuesta.com/questions/view/35/-que-se-entiendepor-participacion-comunitaria.

Mosquera B., Fuentes, Y., Pérez, J. M., \& Pí, A. M. (2011). Comunicación, Educación Popular y Trabajo Social. Revista Digital Sociedad de la Información, 25, 1-9. Recuperado de: http://www.sociedadelainformacion.com

Mujica, N; y Rincón, S. (2010). El concepto de desarrollo. Posiciones teóricas más relevantes. Revista Venezolana de Gerencia, 15(50), 294-320.

Organización Mundial de la Salud [OMS]. (2012). ¿Por qué el tabaco es una prioridad de salud pública? Recuperado de http://www.who.int/tobacco/health_priority/es/index.html.

Pereira, J.M (2003). Comunicación, desarrollo y promoción de la salud: Enfoques, balances y desafíos. La iniciativa de comunicación. Recuperado de http://www. comminit.com/teoriasdecambio/lacth/lasld.html.

Portal, R. (2003). Un estudio de las prácticas comunicativas de los talleres de Transformación Integral de Barrios en la Ciudad de La Habana. (Tesis doctoral). Universidad de La Habana. La Habana.

Portal, R. y Recio, M. (2009). Comunicación y comunidad. La Habana: Félix Varela.

Rebellato, J. L (2000). Antología mínima. La Habana: Editorial Caminos. 
Redondo, P. (2004). Prevención de la enfermedad. Curso de Gestión Local de Salud para Técnicos del Primer Nivel de Atención.

Ruíz, I. M. (2013). Estrategia de Comunicación para la prevención del tabaquismo en los estudiantes de la Universidad Central "Marta Abreu" de Las Villas. (Tesis de Licenciatura). Universidad Central "Martha Abreu" de Las Villas. Santa Clara, Villa Clara, Cuba.

Sánchez, A. (2015). Implementación de la estrategia de comunicación para la prevención de ITS-VIH/SIDA en menores internos en la Escuela de Conducta "Roberto Ambrosio Zamora Machado" de Ciego de Ávila. (Tesis de Licenciatura). Universidad Central "Martha Abreu" de Las Villas. Santa Clara, Villa Clara, Cuba.

Suárez, L. (2011). Paradojas, controversias, discurso y realidad del tabaquismo en Cuba. Revista Cubana Salud Pública 37(1), 5-9.

Varona, T. (2015). Estrategia para contribuir a la disminución del consumo de tabaco en los menores internos en la Escuela de Conducta "Roberto Ambrosio Zamora Machado" de Ciego de Ávila. (Tesis de Licenciatura). Universidad Central "Martha Abreu" de Las Villas. Santa Clara, Villa Clara, Cuba.

Vidal, J. R. (2000). Metodología para elaborar una Estrategia de Comunicación con fines educativos. En J. R. Vidal y M. Alejandro (2004). Comunicación y Educación (pp. 569- 573). Cuba: Caminos.

Villaseñor, M. (2004). Educar para la salud: reto de todos. Educación y Desarrollo, 1(5), 214-221. 